\title{
ESTABILIDAD DEL RENDIMIENTO Y CALIDAD DE GRANO Y TORTILLA DE NUEVOS HÍBRIDOS DE MAÍZ CON VALOR AGREGADO PARA EL SUBTRÓPICO DE MÉXICO
}

\author{
YIELD STABILITY AND GRAIN AND TORTILLA QUALITY OF NEW MAIZE \\ HYBRIDS WITH ADDED VALUE FOR THE SUBTROPICS OF MEXICO
}

\section{Ma. Gricelda Vázquez-Carrillo*, Ricardo E. Preciado-Ortíz², David Santiago-Ramos³, Natalia Palacios-Rojas ${ }^{3}$, Arturo Terrón Ibarra ${ }^{2}$ y Aldo Hernández-Calette ${ }^{1}$}

\begin{abstract}
'Instituto Nacional de Investigaciones Forestales, Agrícolas y Pecuarias (INIFAP), Texcoco, Estado de México, México. ${ }^{2}$ INIFAP, Celaya, Guanajuato, México. ${ }^{3}$ Centro Internacional de Mejoramiento de Maíz y Trigo, Texcoco, Estado de México, México.

*Autor para correspondencia (gricelda_vazquez@yahoo.com)
\end{abstract}

\section{RESUMEN}

El objetivo de este trabajo fue identificar maíces (Zea mays L.) con estabilidad en el rendimiento de grano y potencial para la industria de la masa y la tortilla, mediante la evaluación de la interacción genotipo ambiente de ocho genotipos cultivados en ocho ambientes del subtrópico de México. Durante los ciclos PV-2015 y 2016 se evaluaron maíces con valor agregado: tres de alto contenido de aceite $(\mathrm{H}-327 \mathrm{AO}, \mathrm{H}-388 \mathrm{O}, \mathrm{H}-389 \mathrm{O})$, uno de alta calidad proteínica (H-390 C), un amarillo normal ( $\mathrm{H}-387 \mathrm{~A})$ y tres testigos comerciales (H-377, H-383 C, DK2027Y). Mediante el modelo de regresión de sitios se identificó al híbrido $\mathrm{H}-389 \mathrm{O}$ como uno de los materiales de mayor rendimiento (12.07 $\mathrm{t} \mathrm{ha}^{-1}$ ) y más estable, con buena calidad de grano y producción de tortillas suaves, tanto frescas como almacenadas. En 2015 una mayor precipitación incrementó la acumulación de almidón, la densidad y la dureza del grano. El genotipo H-387 A sobresalió por su alto contenido de carotenoides (12.11 $\mathrm{\mu g} \mathrm{g}^{-1}$ maíz) respecto al testigo amarillo $\left(2.72 \mu \mathrm{g} \mathrm{g}^{-1}\right)$, lo que le confiere propiedades nutracéuticas. El hibrido H-390 C no destacó por su contenido de lisina y triptófano. Aunque poco estables en rendimiento, el resto de los genotipos presentaron características de grano aptas para el procesamiento y rendimiento aceptable de tortillas $\left(1.5 \mathrm{~kg} \mathrm{~kg}^{-1}\right.$ de maíz).

Palabras clave: Zea mays L., Maíces oleosos, calidad proteínica, carotenoides, rendimiento de tortillas.

\section{SUMMARY}

The aim of this work was to identify maize (Zea mays L.) genotypes with stability on grain yield and potential for the masa and tortilla industry, by evaluating the effect of the genotype by environment interaction of eight genotypes grown in eight environments in the subtropics of Mexico. During the spring-summer seasons of 2015 and 2016 we evaluated maize genotypes with added value: three high-oil hybrids ( $\mathrm{H}-327 \mathrm{AO}, \mathrm{H}-388 \mathrm{O}, \mathrm{H}-389 \mathrm{O})$, a quality protein maize hybrid ( $\mathrm{H}-390 \mathrm{C})$, a yellow normal endosperm hybrid $(\mathrm{H}-$ $387 \mathrm{~A})$, and commercial hybrids as control (H-377, H-383 C, DK2027Y). By means of sites regression model, the high-oil hybrid $\mathrm{H}-3890$ was identified as one of the higher grain yield $\left(12.07 \mathrm{t} \mathrm{ha}^{-1}\right)$, more stable, with good grain quality and soft tortillas, both fresh and stored. In 2015 higher precipitation increased the accumulation of starch, grain density and hardness. Carotenoids content of $\mathrm{H}-387 \mathrm{~A}$ (12.11 $\mu \mathrm{g} \mathrm{g}^{-1}$ maize) was higher than yellow control $\left(2.72 \mu \mathrm{g} \mathrm{g}^{-1}\right)$; therefore, it could have nutraceutical potential. The H-390 C hybrid did not highlight for its lysine and tryptophan content. Although the rest of genotypes were not stable on grain yield, they had grains with suitable characteristics for processing and for producing acceptable tortilla yield $\left(1.5 \mathrm{~kg} \mathrm{~kg}^{-1}\right.$ maize).
Index words: Zea mays L., high-oil maize, quality protein, carotenoids, tortilla yield.

\section{INTRODUCCIÓN}

El maíz es el cereal más importante en México en cuanto a producción y consumo, principalmente en forma de tortilla (Vázquez-Carrillo et al., 2015). La tortilla se produce mediante el proceso de nixtamalización, el cual consiste en la cocción de granos de maíz en una solución de agua y cal; el nixtamal se deja reposar, posteriormente se lava y se muele para obtener una masa, la cual se puede deshidratar para producir harinas o se puede utilizar directamente para la elaboración de las tortillas (Santiago-Ramos et al., 2018). Con base en el producto final obtenido se distinguen dos industrias: la de harina nixtamalizada (IHN) e industria de la masa y la tortilla (IMT). La primera se abastece de granos blancos, grandes y duros, con el fin de obtener harinas blancas y evitar el sobrecocimiento y alta absorción de humedad, mientras que la IMT prefiere granos medianos a pequeños y con dureza intermedia, que permitan una alta absorción de agua para obtener rendimientos altos de masa y tortilla (Salinas et al., 2012).

Entre los principales retos que enfrentan ambas industrias está evitar el endurecimiento para aumentar la vida de anaquel e incrementar el valor nutricional de las tortillas. El endurecimiento de las tortillas se resuelve principalmente mediante la adición de gomas. Otra alternativa es el uso de maíces con alto contenido de aceite (ACA), con los cuales también se obtienen tortillas más suaves y con mayor valor nutricional debido a su alto contenido de ácidos grasos insaturados (Vázquez-Carrillo et al., 2015; 2014). Otra opción para incrementar el valor nutricional de las tortillas es el uso de maíces de alta calidad proteínica (QPM) o con alto contenido de carotenoides. El Programa de Mejoramiento Genético de Maíz del Instituto Nacional de Investigaciones Forestales, Agrícolas y Pecuarias (INIFAP) ha trabajado por 
varias décadas en la formación de híbridos de maíz ACA, QPM y pigmentados, con el objetivo no solo de satisfacer la demanda de la industria sino también para ofrecer alternativas en el desarrollo de productos alimenticios con valor agregado alto.

La región del subtrópico en México comprende regiones con altitudes de 1000-2000 metros sobre el nivel del mar (msnm) entre los estados del pacífico, desde Sonora hasta Michoacán y la región de Bajío, una zona que se ha caracterizado por su alta producción de maíz (Ortega-Corona et al., 2015). Los contrastes entre las condiciones climatológicas y edáficas interaccionan con el componente genético del maíz, lo que se expresa en el rendimiento de grano, sus características físicas y su composición química. La respuesta de los genotipos a la interacción genotipo-ambiente se ha evaluado mediante diversos modelos, entre los que destaca el modelo de regresión de sitios (SREG, por sus siglas en inglés), el cual ha demostrado ser efectivo en el análisis de la estabilidad tanto del rendimiento como de las características de calidad del grano y algunas variables de procesamiento (Vázquez-Carrillo et al., 2016). El objetivo de este trabajo fue identificar maíces con estabilidad en el rendimiento de grano y potencial para la IMT mediante la evaluación de la interacción genotipo ambiente de ocho genotipos cultivados en ocho ambientes del subtrópico de México.

\section{MATERIALES Y MÉTODOS}

\section{Material vegetal y sitios experimentales}

Se evaluaron cinco híbridos trilineales de maíz con valor agregado: tres de alto contenido de aceite (ACA), uno de calidad proteínica (QPM) y un amarillo de endospermo normal (Cuadro 1). Los cinco híbridos, de reciente liberación, fueron registrados al inicio de 2018 en el Catálogo Nacional de Variedades Vegetales. Los tres híbridos ACA fueron formados por líneas doble haploides, derivadas de las poblaciones blancas y amarillas del Noroeste y del Bajío (PBN, PAN, PBB y PAB), que fueron formadas a partir de selección recurrente con familias de medios hermanos para incrementar el contenido de aceite en ambas poblaciones, el cual ha sido mayor a 6.0 \% (Ortega-Corona et al., 2015). El H-387 A, que ha destacado por su alta productividad, se desarrolló a través de selección por el método genealógico y cruzas de prueba, formado por dos líneas del CIMMyT y otra del INIFAP. Tres híbridos comerciales (H-377, DK2027Y y H-383 C) se emplearon como testigos.

Todos los híbridos se evaluaron en ocho ambientes del subtrópico. En el ciclo PV-2015 se evaluaron en Pabellón de Arteaga, Aguascalientes (A5, 2050 msnm, siembra 01 de julio), Morelia, Michoacán (M5, 1920 msnm, siembra 29 junio) y dos ambientes en Celaya, Guanajuato (C5, 1760 msnm, siembra 17 junio y G5, siembra, 28 junio). En el ciclo PV-2016 los materiales se sembraron en Morelia, Michoacán (M6, siembra 08 julio), Celaya, Guanajuato en dos ambientes (C6, siembra 31 mayo y G6, siembra 23 julio) y Juventino Rosas, Guanajuato (J6, 2000 msnm, siembra 03 mayo). Las temperaturas y precipitación registradas en ambos años en los ambientes de estudio se muestran en la Figura 1 (INIFAP, 2018).

\section{Rendimiento de grano y características físico-químicas del grano}

El rendimiento de grano (REND en $t \mathrm{ha}^{-1}$ ) se determinó en campo mediante la ecuación reportada por VázquezCarrillo et al. (2016), que considera el peso de la mazorca por parcela a la cosecha, el porcentaje de materia seca del grano ajustado a $14 \%$ de humedad, la proporción de grano

Cuadro 1. Identificación y características de ocho genotipos de maíz cultivados en ocho localidades del subtrópico en México, ciclos PV 2015 y 2016.

\begin{tabular}{lccc}
\hline Nombre & Clave para SREG & Tipo & Color grano \\
\hline Híbridos con valor agregado para el subtrópico & & \\
H-327 AO & AO1 & Alto contenido de aceite (ACA) & Amarillo \\
H-388 O & BO1 & Alto contenido de aceite (ACA) & Blanco \\
H-389 O & BO2 & Alto contenido de aceite (ACA) & Blanco \\
H-390 C & BC1 & Alta calidad proteínica (QPM) & Blanco \\
H-387 A & AN1 & Endospermo normal & Amarillo \\
Testigos comerciales & & & \\
H-377 & BT & Endospermo normal & Blanco \\
H-383 C & CT & Alta calidad proteínica (QPM) & Blanco \\
DK2027Y & AT & Endospermo normal & Amarillo \\
\hline
\end{tabular}

SREG: Método de Regresión de Sitios. 

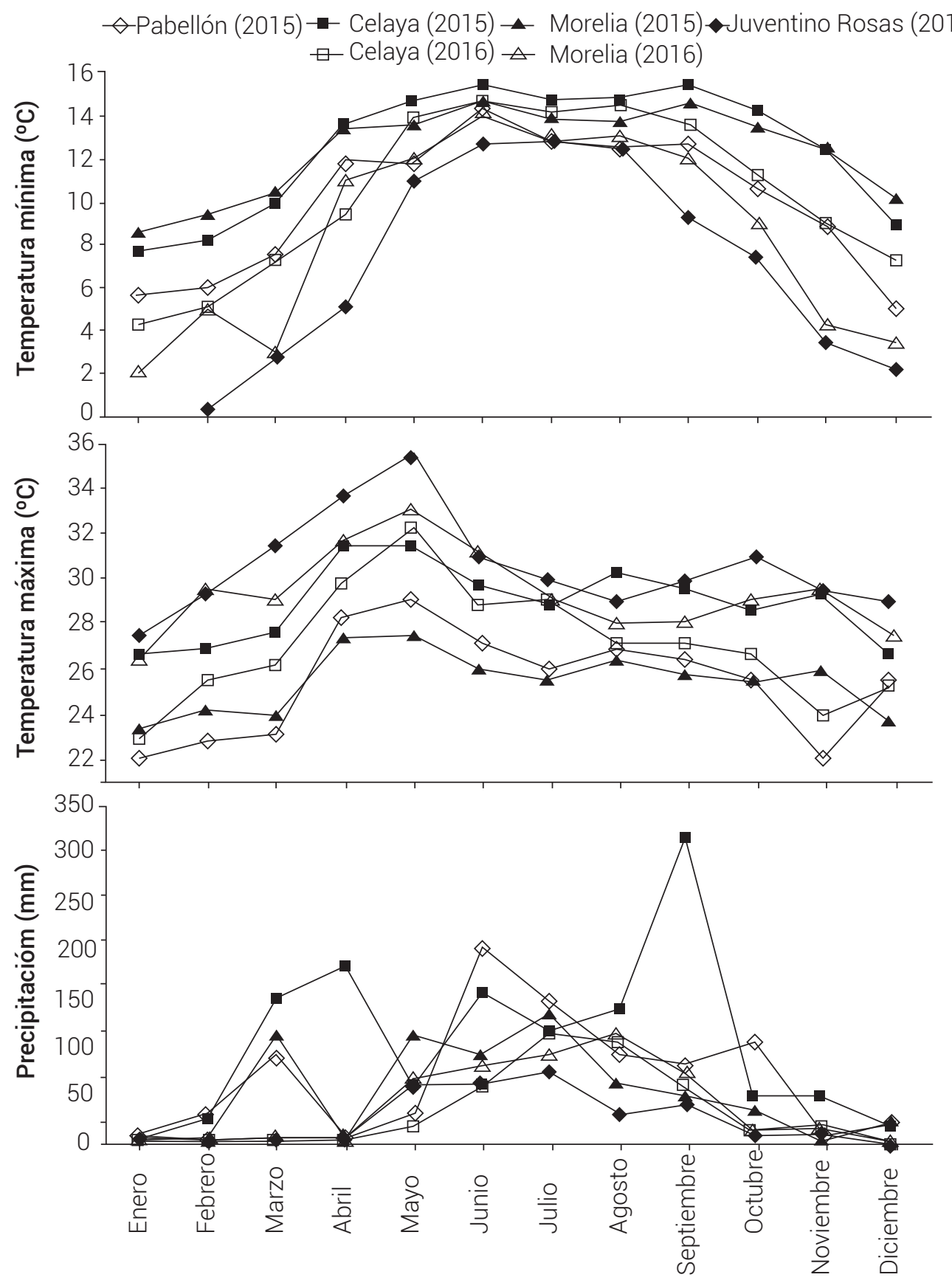

Figura 1. Temperatura mínima y máxima promedio mensual y precipitación de seis localidades del subtrópico de México en 2015 y 2016.

en la mazorca y un factor de corrección que lo extrapola a la superficie de una hectárea. El índice de flotación (IF) se determinó con el método descrito en la norma NMXFF-034/1-SCFI-2002 (Secretaría de Economía, 2002), que consiste en poner 100 granos en una solución de $\mathrm{NaNO}_{3}$ con una densidad de $1.25 \mathrm{~g} \mathrm{~mL}^{-1}$ y contar el número de granos que flotan. Si el número de granos flotantes (IF) es de 1 a 12 los granos se consideran muy duros, si el IF es de 13 a 38 los granos son duros, si el IF es de 39 a 62 los granos son de dureza intermedia, si el IF es de 63 a 87 los granos son suaves, mientras que, si el IF es de 88 a 100 los granos son muy suaves. El peso de 100 granos, así como la composición estructural del grano (pedicelo pericarpio, germen, y endospermo corneo y harinoso) se evaluaron con base en los métodos reportados por Salinas y Vázquez (2006). El peso hectolítrico (PH) y el contenido de aceite, proteína y almidón se determinaron con los métodos 55-10.01, 30-25.01, 46-16.01 y 76-13.01 de la AACC 
International (2018), respectivamente. El contenido de lisina y triptófano se determinó mediante espectroscopía de infrarrojo cercano (NIR) (Rosales et al., 2011). El contenido de carotenoides totales se cuantificó en grano de maíces amarillos con el método descrito por Rosales et al. (2016).

\section{Nixtamalización y elaboración de tortillas}

La nixtamalización y elaboración de las tortillas se realizó por duplicado, siguiendo el método reportado por Vázquez-Carrillo et al. (2016).

\section{Variables del proceso de nixtamalización y calidad de la tortilla}

La pérdida de materia seca (PMS) así como el porcentaje de pericarpio (PR) retenido en el nixtamal se cuantificaron con los métodos descritos por Salinas y Vázquez (2006). La humedad del nixtamal (HN) y de la tortilla se determinó por el método 44-15.02 (AACC International, 2018). El rendimiento de tortilla (RT) se reportó como la cantidad total de tortillas en kg obtenida por cada kg de maíz procesado. La textura de las tortillas se evaluó en términos de la fuerza de ruptura (FR) y elongación, siguiendo la metodología descrita por Vázquez-Carrillo et al. (2015).

\section{Diseño experimental y análisis estadístico}

El diseño experimental en campo fue bloques al azar. Cada unidad experimental consistió de dos surcos de cinco $\mathrm{m}$ de longitud y $0.66 \mathrm{~m}$ de ancho, con dos repeticiones. Los datos obtenidos se sometieron a análisis de varianza (ANOVA) individual y conjunto y se realizó la comparación de medias con la prueba de Tukey $(P \leq 0.05)$. Las variables con interacción genotipo-ambiente (IGA) significativa fueron sometidas a un análisis de regresión de sitios (SREG) para obtener un gráfico de componentes principales (CP) que permite visualizar tanto a los genotipos como a los ambientes. La interpretación de los gráficos se realizó sólo si los dos primeros CP explicaron al menos $75 \%$ de la IGA (Lozano-Ramírez et al., 2015).

\section{RESULTADOS Y DISCUSIÓN}

\section{Rendimiento y características físicas del grano}

En rendimiento, los genotipos H-388 O (BO1) y DK2027Y (AT) mostraron los mayores rendimientos $\left(>12.8 \mathrm{t} \mathrm{ha}^{-1}\right)$, mientras que $\mathrm{H}$-327 AO (AO1), $\mathrm{H}-383 \mathrm{C}(\mathrm{CT})$ y H-377 (BT) tuvieron los más bajos (<10.05 t ha-1) (Cuadro 2); sin embargo, en todos los casos el rendimiento fue superior a la media nacional, que al cierre de mayo de 2018 fue de $5.1 \mathrm{t}$ $\mathrm{ha}^{-1}$ (SIAP, 2018).

La Figura 2 muestra los gráficos del análisis SREG para las variables cuyos dos primeros CP explicaron al menos $75 \%$ de la IGA. El genotipo más estable fue H-389 O (BO2) (Figura 2A), que también tuvo un REND estadísticamente igual a H-387 A (AN1). Respecto a los ambientes, es sabido que ambientes con vectores largos discriminan mejor entre genotipos; en ese sentido, la mayor variabilidad entre genotipos se observó en G5, mientras que en J6 y G6 los híbridos presentaron REND similares.

Al trazar un polígono entre los genotipos más alejados del origen y líneas perpendiculares que van del origen a cada lado del polígono, los ambientes con respuesta similar se agrupan en mega-ambientes y se pueden identificar genotipos que se adaptan a los mismos (Karimizadeh et al., 2013). Así, en REND se formaron dos mega-ambientes; en el primero se concentraron A5, M6, G6 y C6, ambientes con poca precipitación y temperatura altas (Figura 1), donde el genotipo DK2027Y (AT) fue el mejor adaptado a éste. Por otro lado, el segundo mega-ambiente comprendió M5, G5, C5 y J6, que presentaron buena precipitación (Figura 1) y el genotipo con mejor adaptación y mayor rendimiento

Cuadro 2. Medias de rendimiento y calidad de grano de ocho genotipos de maíz cultivados en ocho ambientes del subtrópico en México, ciclos PV 2015 y 2016.

\begin{tabular}{lcccccccc}
\hline Genotipo & Rendimiento $\left(\mathrm{t} \mathrm{ha}^{-1}\right)$ & IF & Textura $^{£}$ & PCG $(\mathrm{g})$ & PH $\left(\mathrm{kg} \mathrm{hL}^{-1}\right)$ & Aceite $(\%)$ & Almidón (\%) & Proteína (\%) \\
\hline H-327 AO & $8.96 \mathrm{c}$ & $34 \mathrm{a}$ & Duro & $32.47 \mathrm{~d}$ & $77.68 \mathrm{de}$ & $6.79 \mathrm{a}$ & $65.84 \mathrm{e}$ & $10.37 \mathrm{a}$ \\
$\mathrm{H}-388 \mathrm{O}$ & $12.86 \mathrm{a}$ & $30 \mathrm{~b}$ & Duro & $31.59 \mathrm{e}$ & $77.50 \mathrm{e}$ & $6.54 \mathrm{a}$ & $67.83 \mathrm{~d}$ & $8.93 \mathrm{~d}$ \\
$\mathrm{H}-390 \mathrm{C}$ & $10.81 \mathrm{bc}$ & $24 \mathrm{c}$ & Duro & $33.00 \mathrm{~d}$ & $78.29 \mathrm{~cd}$ & $5.52 \mathrm{c}$ & $68.62 \mathrm{c}$ & $9.46 \mathrm{bc}$ \\
$\mathrm{H}-389 \mathrm{O}$ & $12.07 \mathrm{ab}$ & $23 \mathrm{c}$ & Duro & $29.30 \mathrm{f}$ & $78.05 \mathrm{cde}$ & $5.78 \mathrm{~b}$ & $70.43 \mathrm{a}$ & $9.77 \mathrm{~b}$ \\
DK2027Y & $12.80 \mathrm{a}$ & $21 \mathrm{~d}$ & Duro & $38.68 \mathrm{a}$ & $78.62 \mathrm{bc}$ & $5.13 \mathrm{~d}$ & $69.04 \mathrm{bc}$ & $9.15 \mathrm{~cd}$ \\
H-383 C & $9.42 \mathrm{c}$ & $18 \mathrm{e}$ & Duro & $31.72 \mathrm{e}$ & $79.25 \mathrm{~b}$ & $4.75 \mathrm{e}$ & $70.78 \mathrm{a}$ & $8.02 \mathrm{e}$ \\
H-377 & $10.05 \mathrm{c}$ & $16 \mathrm{f}$ & Duro & $35.23 \mathrm{~b}$ & $78.51 \mathrm{c}$ & $5.07 \mathrm{~d}$ & $69.51 \mathrm{~b}$ & $9.49 \mathrm{~b}$ \\
H-387 A & $12.17 \mathrm{ab}$ & $5 \mathrm{~g}$ & Muy & $34.49 \mathrm{c}$ & $82.68 \mathrm{a}$ & $4.34 \mathrm{f}$ & $68.80 \mathrm{c}$ & $9.06 \mathrm{~d}$ \\
\hline
\end{tabular}

IF: índice de flotación; £|F: 0-12, Muy Duro; £IF: 13-36, Duro; PCG: peso de cien granos; PH: peso hectolítrico. Medias con letras iguales no son estadísticamente diferentes (Tukey, $\mathrm{P} \leq 0.05$ ). 

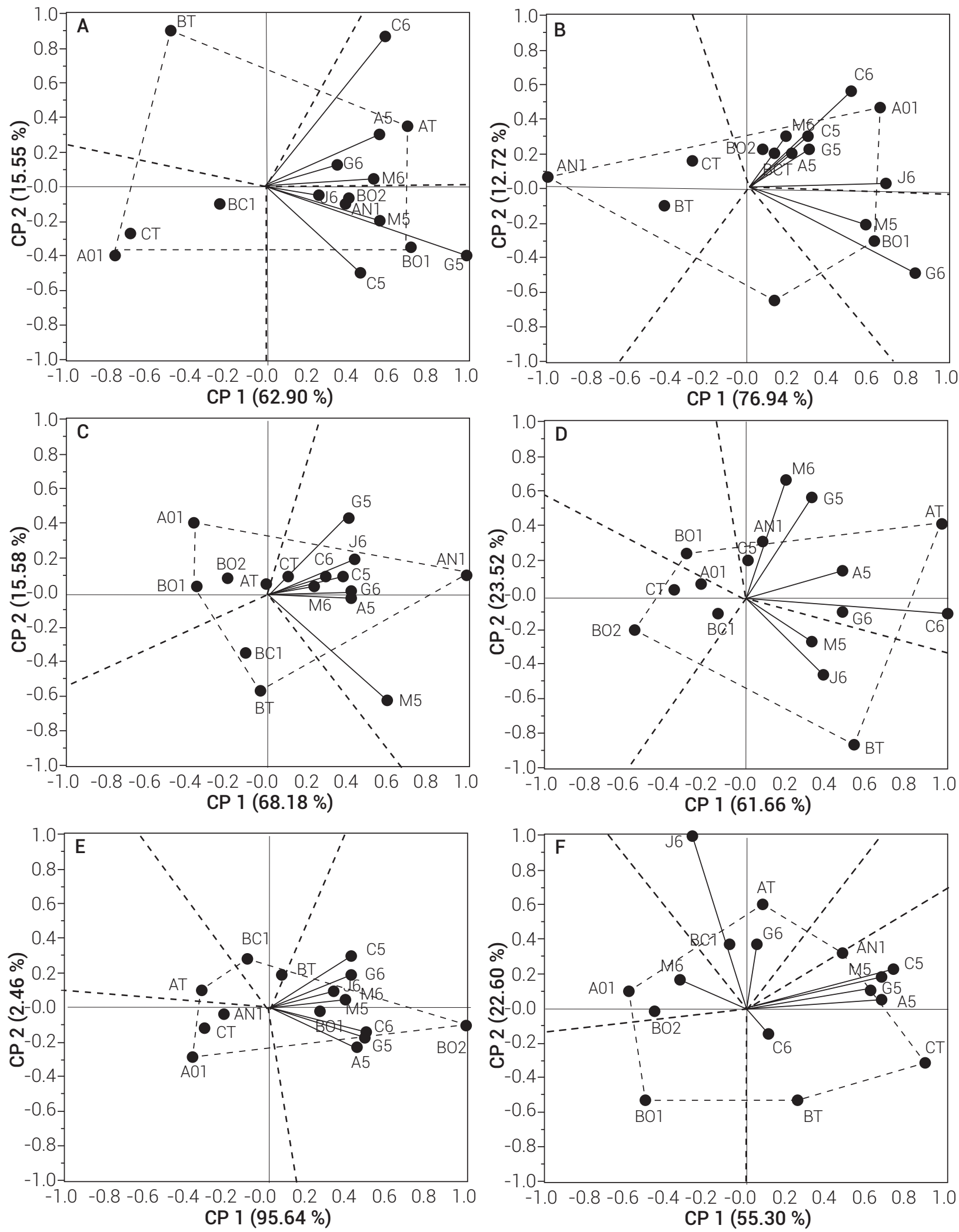

Figura 2. Gráficas biplot del análisis SREG para rendimiento (A), índice de flotación (B), peso hectolítrico (C), peso de 100 granos (D), pericarpio (E) y almidón (F) de ocho genotipos de maíz evaluados en ocho ambientes de los ciclos PV-2015 y 2016 del subtrópico de México. 
fue H-388 O (BO1). Este último comportamiento es el más deseable: aumentar el contenido de aceite sin disminuir el rendimiento; entre los factores que afectan este resultado están la sequía y la condición del suelo (Torres-Morales et al., 2010). En ese sentido, las buenas condiciones de precipitación en esos ambientes influyeron positivamente en la acumulación tanto de almidón como de aceite.

Los híbridos de alto contenido de aceite $\mathrm{H}-327$ AO (A01) y H-388 O (BO1) tuvieron los valores más altos de IF (Figura 2B) en la mayoría de los ambientes, mientras que el híbrido H-387 A (AN1) fue el único de grano muy duro y el más estable en su dureza (Figura 2B). De acuerdo con la clasificación de la norma NMX-FF-034/1-SCFI-2002 (Secretaría de Economía, 2002), todos los genotipos tuvieron granos duros y muy duros (Cuadro 2), por lo que son aptos para su transformación, tanto en harinas nixtamalizadas $(\mathrm{H}-390 \mathrm{C}$ y H-387A), como en masa fresca por la IMT (los cinco nuevos híbridos). Se observó que en 2015 se obtuvieron granos más duros que en 2016; esto pudo ser consecuencia de una mayor y mejor distribución de la precipitación durante todas las etapas de crecimiento de la planta (Figura 1), lo que influyó positivamente durante el periodo de llenado de grano. Vázquez-Carrillo et al. (2016) observaron resultados similares respecto al efecto positivo de la precipitación en la acumulación de almidón en el grano y su efecto en la dureza.

La gráfica de PH (Figura 2C) mostró el efecto contrario a la de IF, ya que existe alta correlación entre ambas variables $(r=-0.68, P \leq 0.05)$, por lo que los granos más duros tienden a ser más densos (Narváez-González et al., 2006). En PH el genotipo más estable fue el testigo DK2027Y (Figura 2C). Todos los genotipos tuvieron un $\mathrm{PH} \geq 74 \mathrm{~kg} \mathrm{hL}^{-1}$ (Cuadro 2), por lo que son aptos para el procesamiento por ambas industrias (NMX-FF-034/1-SCFI-2002) (Secretaria de Economía, 2002).

Salinas et al. (2010) reportan que los granos grandes tienen un PCG mayor a $38 \mathrm{~g}$, los medianos entre 33 y $38 \mathrm{~g}$, en tanto que los pequeños presentan valores menores a 33 g. Con base en esa clasificación sólo el testigo amarillo (DK2027Y) tuvo granos grandes, el resto tuvo granos de tamaño mediano a pequeño (Cuadro 2). La mayoría de los ambientes propició un mayor tamaño de grano en el testigo DK2027Y y granos pequeños en el resto de los híbridos (Figura 2D).

En general, el porcentaje de pedicelo y germen fue alto en los genotipos de alto contenido de aceite, comparado con los testigos de endospermo normal (Cuadro 3). Torres-Morales et al. (2010) reportaron que al seleccionar mazorcas por contenido de aceite también aumenta el tamaño del germen. Coincidente con su bajo contenido de aceite en el genotipo H-389 O la IGA propició un aumento del contenido de pedicelo y pericarpio en vez del germen. El gráfico de contenido de pericarpio (Figura 2E) mostró que el genotipo más estable en esta variable fue $\mathrm{H}$-388 $\mathrm{O}$ (B01), y que todos los ambientes influyeron en que el genotipo H-389 O (BO2) presentara mayor contenido de esta estructura, lo que se reflejó en el promedio general (Cuadro 3).

En México, la IHN prefiere maíces con un contenido de pericarpio menor al 5.5 \% (Salinas et al., 2012), debido a que la mayor parte de esta estructura se remueve durante el procesamiento para obtener harinas blancas; el genotipo BO2 no cumple esta especificación. En cambio, todos los híbridos pueden ser procesados por la IMT debido a que en esta industria es deseable retener la mayor cantidad posible de pericarpio hidrolizado, el cual actúa como hidrocoloide mejorando la textura de las tortillas (Santiago-Ramos et al., 2018). La IHN procesa maíces con proporciones de germen menor al 13.0 \% (Salinas et al., 2012), ya que su elevado contenido de aceite dificulta la molienda del nixtamal y reduce la vida de anaquel de las harinas. Por lo que los genotipos ACA no son aptos para la IHN. De acuerdo a sus componentes estructurales, todos los híbridos son adecuados para la IMT. Aunque se ha sugerido que el aumento de la calidad proteínica conlleva a un aumento en la vitreosidad del endospermo (Ignjatovic et al., 2013) no se encontraron diferencias importantes en contenido de endospermo harinoso y córneo entre genotipos.

\section{Componentes químicos del grano}

El mejoramiento genético del maíz enfocado en los componentes químicos se ha concentrado en aumentar el contenido de almidón, de aceite, y la calidad de la proteína; sin embargo, el aumento en el contenido de uno de los componentes puede afectar la acumulación de los demás. Lambert et al. (1998) mencionaron que la acumulación de aceite y almidón son mutuamente excluyentes, debido a que acumular una determinada cantidad de aceite requiere 2.25 veces más energía que acumular la misma cantidad de almidón o proteína. Los genotipos con menor densidad tuvieron un menor contenido de almidón (Cuadro 2), donde los de ACA H-327 AO (AO1) y H-389 O (BO2) fueron los más estables en este componente (Figura 2F). En concordancia con los resultados de IF y PH, en 2015 todos los genotipos registraron un mayor contenido de almidón que en 2016, producto de una mayor acumulación. Esto se debe a que en 2016 las temperaturas altas y la menor precipitación interrumpen la división celular, el metabolismo de los azúcares y por tanto la biosíntesis del almidón en el endospermo, como lo reportaron Vázquez-Carrillo et al. (2016). 
Cuadro 3. Medias de la composición estructural de ocho genotipos de maíz cultivados en ocho ambientes del subtrópico en México, ciclos PV 2015 y 2016.

\begin{tabular}{lccccc}
\hline Genotipo & Pedicelo (\%) & Pericarpio (\%) & Germen (\%) & EC (\%) & EH (\%) \\
\hline H-327 AO & $1.85 \mathrm{a}$ & $3.93 \mathrm{f}$ & $14.57 \mathrm{a}$ & $48.84 \mathrm{ab}$ & $30.82 \mathrm{~b}$ \\
$\mathrm{H}-388 \mathrm{O}$ & $1.83 \mathrm{a}$ & $5.30 \mathrm{~b}$ & $12.86 \mathrm{~b}$ & $44.57 \mathrm{~b}$ & $35.44 \mathrm{ab}$ \\
$\mathrm{H}-390 \mathrm{C}$ & $1.35 \mathrm{c}$ & $4.54 \mathrm{~d}$ & $13.05 \mathrm{ab}$ & $45.34 \mathrm{~b}$ & $35.73 \mathrm{ab}$ \\
H-389 O & $1.92 \mathrm{a}$ & $6.82 \mathrm{a}$ & $12.72 \mathrm{bc}$ & $45.41 \mathrm{~b}$ & $33.13 \mathrm{ab}$ \\
DK2027Y & $1.43 \mathrm{bc}$ & $4.02 \mathrm{f}$ & $12.84 \mathrm{~b}$ & $43.18 \mathrm{~b}$ & $38.53 \mathrm{a}$ \\
H-383 C & $1.56 \mathrm{c}$ & $4.04 \mathrm{ef}$ & $11.49 \mathrm{bc}$ & $48.95 \mathrm{ab}$ & $33.96 \mathrm{ab}$ \\
H-377 & $1.38 \mathrm{c}$ & $4.87 \mathrm{c}$ & $11.16 \mathrm{c}$ & $46.97 \mathrm{ab}$ & $35.62 \mathrm{ab}$ \\
H-387 A & $1.29 \mathrm{c}$ & $4.25 \mathrm{e}$ & $12.06 \mathrm{bc}$ & $51.63 \mathrm{a}$ & $30.77 \mathrm{~b}$ \\
\hline
\end{tabular}

EC: endospermo corneo; EH: endospermo harinoso. Medias con letras iguales no son estadísticamente diferentes (Tukey, P $\leq 0.05)$.

Los híbridos ACA H-327 AO (A01) y H-388 O (BO1) tuvieron el mayor contenido de aceite, no así el híbrido H-389 O (BO2), el cual tuvo un contenido de aceite inferior a lo esperado para este tipo de maíz (> 6.0 \%) (Cuadro 2). Estos resultados indican que el híbrido $\mathrm{H}-389 \mathrm{O}$ (BO2) fue altamente afectado por la IGA. Torres-Morales et al. (2010) reportaron que la sequía y las condiciones del suelo son factores que influyen negativamente en la acumulación del aceite. El elevado contenido de germen y consecuentemente de aceite de los híbridos $\mathrm{H}$-327AO y H-3880 limita su aprovechamiento por la IHN; por el contrario, para la IMT el procesamiento de maíces ACA les permitirá ofrecer a los consumidores tortillas más suaves (Vázquez-Carrillo et al., 2016) y con mejor valor nutricional, debido a sus ácidos grasos insaturados. En el contenido de proteína no se observó una tendencia clara entre tipos de maíz. El genotipo ACA H-327 AO (AO1) presentó el mayor contenido mientras que el testigo QPM el más bajo (Cuadro 2). En promedio, los ambientes del año 2016 propiciaron un mayor contenido de carotenoides (Cuadro 4).

Al ser éstos parte del metabolismo secundario de las plantas es posible que su mayor síntesis se haya dado como respuesta al estrés generado en la planta por las temperaturas más altas registradas en ese año. El contenido de carotenoides cuantificado en el genotipo DK2027Y fue similar al reportado por Corrales et al. (2016) (2.61 a 5.56 $\mu \mathrm{g} \mathrm{g}^{-1}$ ), mientras que los genotipos $\mathrm{H}-327 \mathrm{AO}$ y H-387 A tuvieron mayor contenido de carotenoides respecto a lo reportado por los mismos autores. Por su alto contenido de carotenoides, el genotipo H-387 A puede ser un maíz con características nutracéuticas excepcionales, debido a que estos compuestos proveen protección contra el estrés oxidativo, incrementan la actividad antimutagénica, previenen el cáncer y reducen el riesgo de la degeneración macular asociada con la edad (Corrales et al., 2016).

En cuanto a la calidad proteica, representada por el contenido de lisina y triptófano, se observaron diferencias significativas en su contenido entre genotipos y entre ambientes, pero no por efecto de la IGA. De acuerdo con Twumasi-Afriyie et al. (2016), para que un maíz sea considerado como QPM debe tener en grano al menos 0.075 $\%$ de triptófano y $0.35 \%$ de lisina; sin embargo, esto no se encontró en los genotipos evaluados y considerados como tal (Cuadro 4). Estos resultados pueden explicarse por una posible contaminación por polinización abierta, pues algunas variedades QPM son muy susceptibles a tal contaminación y pierden su característica de calidad (Teklewold et al., 2015).

\section{Variables del proceso de nixtamalización y calidad de la tortilla}

La humedad de nixtamal osciló entre 46 y $52 \%$ (Cuadro 5), valores similares a los reportados por Salinas et al. (2012).

Los genotipos de alto contenido de aceite y el QPM retuvieron más agua ( $H N \geq 49 \%$ ), y este comportamiento se mantuvo en la masa y en la tortilla (Cuadro 5), debido a que la menor dureza del grano y su baja densidad contribuyeron a una mayor difusión del agua hacia el interior del mismo.

La PMS es un indicador de la contaminación generada durante el procesamiento por desechos del nejayote; esta genera pérdidas económicas disminuyendo el rendimiento de masa y tortillas y su calidad por pérdidas de almidón y componentes del pericarpio. Por esta razón, una PMS < 5.0 \% (Secretaría de Economía, 2002) se considera ideal, y como tal se presentó en el procesamiento de todos los genotipos (Cuadro 5). Las muestras del genotipo H-389 0 (BO2) provenientes de la mayoría de los ambientes tuvieron una mayor PMS (Figura 3A), excepto en C6; esto se debió al reducido tamaño de sus granos (PCG: $29.3 \mathrm{~g}$ ) y al 
Cuadro 4. Medias de contenido de carotenoides, lisina y triptófano en grano de ocho genotipos de maíz cultivados en ocho ambientes del subtrópico en México, ciclos PV 2015 y 2016.

\begin{tabular}{|c|c|c|c|c|c|c|c|}
\hline Ambiente & $\begin{array}{l}\text { Carotenoides } \\
\left(\mu \mathrm{g} \mathrm{g}^{-1} \text { maíz) }\right.\end{array}$ & Triptófano (\%) & Lisina(\%) & Genotipo & $\begin{array}{l}\text { Carotenoides } \\
\left(\mu \mathrm{g} \mathrm{g}^{-1} \text { maíz }\right)\end{array}$ & Triptófano (\%) & Lisina (\%) \\
\hline C6 & $10.49 \mathrm{a}$ & $0.050 \mathrm{bc}$ & $0.285 a b$ & $\mathrm{H}-327 \mathrm{AO}$ & $6.91 b$ & $0.056 a b c$ & $0.282 \mathrm{bcd}$ \\
\hline G6 & $8.84 a b$ & $0.060 \mathrm{a}$ & $0.310 a$ & $\mathrm{H}-388 \mathrm{O}$ & & $0.049 \mathrm{~d}$ & $0.264 d$ \\
\hline M6 & $7.49 b$ & $0.054 a b c$ & $0.301 \mathrm{a}$ & $\mathrm{H}-390 \mathrm{C}$ & & $0.058 \mathrm{abc}$ & $0.316 a$ \\
\hline G5 & $7.37 \mathrm{~b}$ & 0.058 a & $0.269 \mathrm{bc}$ & H-389 O & & $0.049 \mathrm{~d}$ & $0.271 \mathrm{c}$ \\
\hline J6 & $6.58 \mathrm{bc}$ & $0.056 a b c$ & $0.306 a$ & DK2027Y & $2.72 \mathrm{c}$ & $0.053 \mathrm{~cd}$ & $0.280 b$ \\
\hline C5 & $6.18 \mathrm{bc}$ & $0.056 a b$ & $0.259 \mathrm{bc}$ & H-383 C & & $0.061 \mathrm{a}$ & $0.307 a b$ \\
\hline M5 & $6.17 \mathrm{bc}$ & $0.049 \mathrm{c}$ & $0.252 \mathrm{c}$ & $\mathrm{H}-377$ & & $0.056 \mathrm{abc}$ & $0.301 \mathrm{abc}$ \\
\hline A5 & $4.34 \mathrm{c}$ & $0.056 \mathrm{ab}$ & $0.301 \mathrm{a}$ & $\mathrm{H}-387 \mathrm{~A}$ & $12.11 \mathrm{a}$ & $0.054 \mathrm{bcd}$ & $0.269 \mathrm{~d}$ \\
\hline
\end{tabular}

Medias con letras iguales no son estadísticamente diferentes (Tukey, $\mathrm{P} \leq 0.05$ ).

Cuadro 5. Medias de variables del proceso de nixtamalización y calidad de tortilla de ocho genotipos de maíz cultivados en ocho localidades del subtrópico en México, ciclos PV 2015 y 2016.

\begin{tabular}{llllllll}
\hline Genotipo & HN (\%) & PMS (\%) & PR (\%) & FR (N) & Elongación $(\mathrm{mm})$ & HT (\%) & RT $\left(\mathrm{kg} \mathrm{kg} \mathrm{maíz}^{-1}\right)$ \\
\hline H-327 AO & $48.56 \mathrm{bc}$ & $3.31 \mathrm{c}$ & $50.02 \mathrm{bc}$ & $2.01 \mathrm{c}$ & $7.21 \mathrm{~d}$ & $41.66 \mathrm{~b}$ & $1.46 \mathrm{a}$ \\
H-388 O & $48.98 \mathrm{~b}$ & $3.65 \mathrm{~b}$ & $45.55 \mathrm{c}$ & $2.43 \mathrm{abc}$ & $8.04 \mathrm{abcd}$ & $43.93 \mathrm{a}$ & $1.49 \mathrm{a}$ \\
H-390 C & $48.85 \mathrm{~b}$ & $3.35 \mathrm{c}$ & $53.12 \mathrm{ab}$ & $2.78 \mathrm{a}$ & $8.64 \mathrm{a}$ & $43.47 \mathrm{ab}$ & $1.49 \mathrm{a}$ \\
H-389 O & $51.57 \mathrm{a}$ & $3.95 \mathrm{a}$ & $49.20 \mathrm{bc}$ & $2.51 \mathrm{a}$ & $8.20 \mathrm{abc}$ & $43.99 \mathrm{a}$ & $1.49 \mathrm{a}$ \\
DK2027Y & $46.89 \mathrm{~cd}$ & $2.75 \mathrm{f}$ & $55.32 \mathrm{a}$ & $2.48 \mathrm{ab}$ & $8.51 \mathrm{ab}$ & $42.75 \mathrm{ab}$ & $1.52 \mathrm{a}$ \\
H-383 C & $48.45 \mathrm{bc}$ & $2.82 \mathrm{e}$ & $52.54 \mathrm{ab}$ & $2.44 \mathrm{abc}$ & $7.71 \mathrm{bcd}$ & $44.05 \mathrm{a}$ & $1.53 \mathrm{a}$ \\
H-377 & $49.41 \mathrm{~b}$ & $3.33 \mathrm{c}$ & $53.98 \mathrm{ab}$ & $2.05 \mathrm{bc}$ & $7.51 \mathrm{~cd}$ & $43.31 \mathrm{ab}$ & $1.49 \mathrm{a}$ \\
H-387 A & $46.38 \mathrm{~d}$ & $3.14 \mathrm{~d}$ & $46.95 \mathrm{c}$ & $2.66 \mathrm{a}$ & $8.23 \mathrm{abc}$ & $41.73 \mathrm{~b}$ & $1.44 \mathrm{a}$ \\
\hline
\end{tabular}

HN: humedad de nixtamal, PMS: pérdida de materia seca, PR: pericarpio retenido, FR: fuerza de ruptura medida en Newtons, HT: humedad de tortilla (2h), RT: rendimiento de tortilla. Medias con letras iguales no son estadísticamente diferentes (Tukey, $\mathrm{P} \leq 0.05)$.

elevado contenido de pericarpio (6.82 \%) (Figura 2E). El PR y la PMS se correlacionaron negativamente $(r=-0.4, P \leq$ 0.05), ya que la mayor PMS se atribuye al pericarpio perdido. Es importante que parte del pericarpio hidrolizado se conserve después de la nixtamalización, pues contribuye en la retención de agua y hace más elásticas las tortillas (Vázquez-Carrillo et al., 2015). Todos los genotipos retuvieron entre el 45 y el $55 \%$ del pericarpio, lo cual supera el valor mínimo de $30 \%$ esperado por la IMT (Salinas et al., 2010).

Con los maíces H-377 (BT), H-390 C (BC1) y H-383 C (CT) se produjeron las tortillas con mayor humedad recién elaboradas (Cuadro 5) y después de almacenadas (Figura 3B). Con el testigo amarillo DK2027Y se produjeron las tortillas con menor humedad (Figura 3B). El RT de todos los genotipos fue similar y cercano a $1.5 \mathrm{~kg} \mathrm{~kg}^{-1}$ de maíz procesado, valor que se considera aceptable para la IMT. Las tortillas frescas de los genotipos de ACA tuvieron tortillas ligeramente más duras que las del resto (Cuadro 5), pero $24 \mathrm{~h}$ después de almacenadas estas tortillas fueron las más suaves (datos no mostrados). Este comportamiento se debe a que en maíces con alto contenido de aceite los ácidos grasos libres interaccionan con el almidón gelatinizado, formando complejos amilosa-lípido, lo que inhibe la retrogradación provocando que las tortillas sean menos duras durante el almacenamiento (Vázquez-Carrillo et al., 2015).

\section{CONCLUSIONES}

Con ayuda del modelo SREG se pudo identificar que el genotipo ACA H-389 O (BO2) presentó la mayor estabilidad a través de todos los ambientes y tuvo un rendimiento de grano alto, similar al testigo DK2027Y. Otro genotipo estable pero solo en ambientes con buena precipitación fue el H-388 O (B01). Todos los híbridos ACA cumplieron con los requerimientos de calidad de la industria de masa fresca para tortillas, las cuales en almacenamiento fueron más suaves que las de los híbridos de endospermo normal. Para la IHN se recomiendan los híbridos H-390C y H-387A. La distribución de la precipitación durante todo el periodo 

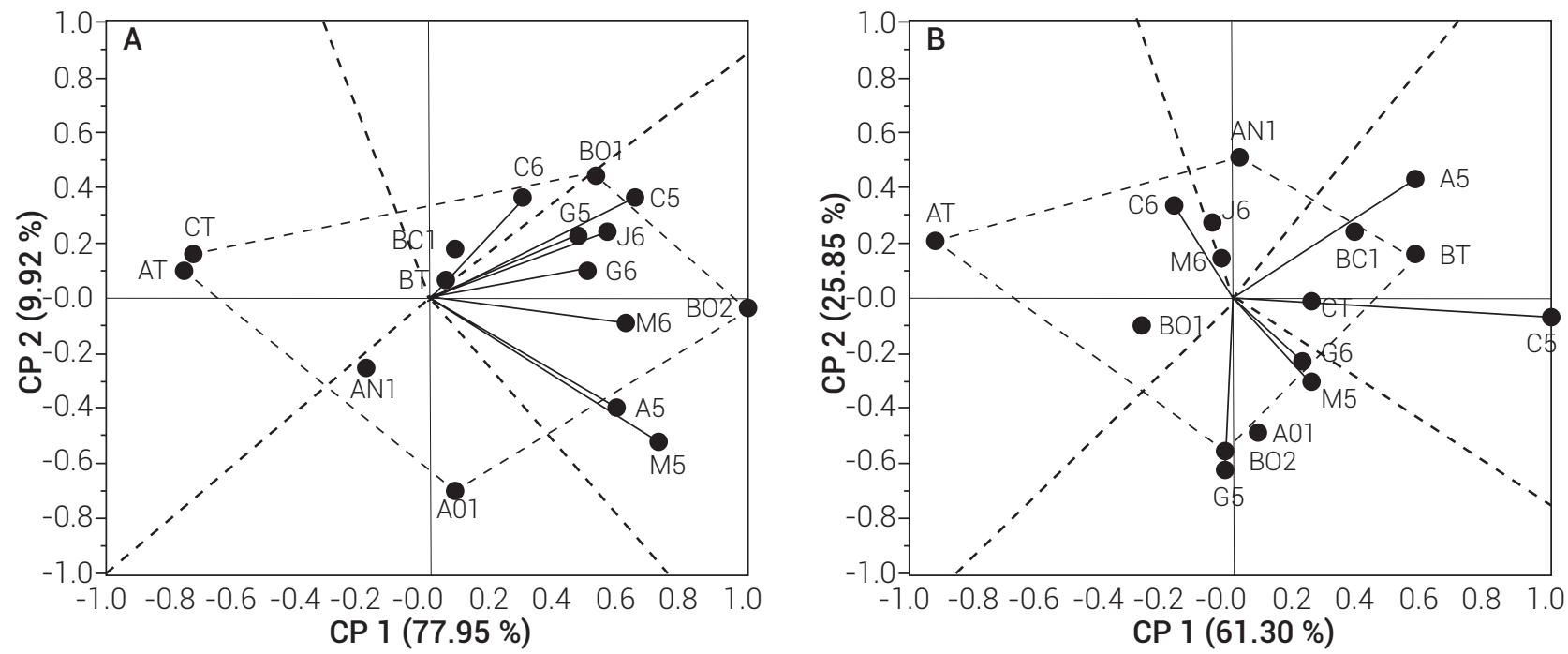

Figura 3. Gráficas biplot del análisis SREG para pérdida de materia seca (A) y humedad de tortilla a las $24 \mathrm{~h}$ (B) de ocho genotipos de maíz evaluados en ocho ambientes de los ciclos PV-2015 y 2016 del subtrópico de México.

de desarrollo de la planta fue el principal factor que influyó en una mayor dureza, densidad y acumulación de almidón. El genotipo amarillo H-387 A destacó por su alto contenido de carotenoides, lo cual puede tener una ventaja en su comercialización por sus propiedades nutracéuticas.

\section{BIBLIOGRAFÍA}

AACC International (2018) Approved Methods of Analysis 11 th Ed. American Association of Cereal Chemists International. St. Paul, MN, USA. Disponible en http://methods.aaccnet.org/.

Corrales B. A., E. Cuevas R., J. Gutiérrez U., E. Milán N., C. Reyes M., J. Milán C. and S. Mora R. (2016) Carotenoid composition and antioxidant activity of tortillas elaborated from pigmented maize landrace by traditional nixtamalization or lime cooking extrusion process. Journal of Cereal Science 69:64-70.

Ignjatovic M., D. Dragana, M. Kostadinovic, G. Stankovic, K. Markovic, J. Vancetovic, S. Bozinovic and V. Andjelkovic (2013) Biochemical and agronomic performance of quality protein maize hybrids adapted to temperate regions. Maydica 58:311-317.

INIFAP, Instituto Nacional de Investigaciones Forestales, Agrícolas y Pecuarias (2018) Red Nacional de Estaciones Estatales Agro-climatológicas. Disponible en: http://clima.inifap. gob.mx/red- clima (Junio 2018)

Karimizadeh R., M. Mohammadi and N. Sabaghmia (2013) Site regression biplot analysis for matching new improved lentil genotypes into target environment. Journal of Plant Physiology and Breeding 3:51-65.

Lambert R. J., D. E. Alexander and Z. J. Han (1998) A high oil pollinator enhancement of kernel oil and effects on grain yields of maize hybrids. Agronomy Journal 90:211-215.

Lozano-Ramírez A., A Santacruz-Varela, F San-Vicente-García, J. Crossa, J. Burqueño y J. D. Molina G. (2015) Modelación de la interacción genotipo $x$ ambiente en rendimiento de híbridos de maíz blanco en ambientes múltiples. Revista Fitotecnia Mexicana 38:337347.

Narváez-González E. D., J. D. Figueroa-Cárdenas, S. Taba, E. Castaño-Tostado, R. A. Martínez- Peniche and F. Rincón-Sánchez (2006) Relationships between the microstructure, physical features, and chemical composition of different maize accessions from Latin America. Cereal Chemistry 83:595-604.

Ortega-Corona A., R. Picón-Rico, R. E. Preciado-Ortiz, A. D. Terrón-Ibarra, M. J.
Guerrero-Herrera, S. García-Lara and S. O. Serna-Saldivar (2015) Selection response for oil content and agronomic performance in four subtropical maize populations. Maydica 60:1-8.

Rosales A., E. Agama-Acevedo, L. A. Bello-Pérez, R. Gutiérrez-Dorado and N. Palacios-Rojas (2016) Effect of traditional and extrusion nixtamalization on carotenoid retention in tortillas made from provitamin A biofortified maize (Zea mays L.). Journal of Agricultural and Food Chemistry 64:8289-8295.

Rosales A., L. Galicia, E. Oviedo, C. Islas and N. Palacios R. (2011) Near-infrared reflectance spectroscopy (NIRS) for protein, tryptophan, and lysine evaluation in Quality Protein Maize (QPM) breeding programs. Journal of Agricultural and Food Chemistry 59:10781-10786.

Salinas M. Y. y M. G. Vázquez C. (2006) Metodologías de Análisis de Calidad Nixtamalera-Tortillera en Maíz. INIFAP. Ciudad de México, México. Folleto Técnico No. 23. $91 p$

Salinas M. Y., N. O. Gómez M., J. E. Cervantes M., M. Sierra M., A. Palafox C., E. Betanzos M. y B. Coutiño E. (2010) Calidad nixtamalera y tortillera en maíces del trópico húmedo y sub-húmedo de México. Revista Mexicana de Ciencias Agrícolas 1:509-523.

Salinas M. Y., M. G. Vázquez C., G. A. Velázquez C. y J. Soria R. (2012) Esquema de Selección de Maíces con Calidad para Elaborar MasaTortilla y Harinas Nixtamalizadas: Caso Estado de México. INIFAP. Texcoco, México. Folleto Técnico No. 4268 p.

Santiago-Ramos D., J. D. Figueroa-Cárdenas, R. M. Mariscal-Moreno, A. Escalante-Aburto, N. Ponce-García and J. J. Veles-Medina (2018) Physical and chemical changes undergone by pericarp and endosperm during corn nixtamalization-A review. Journal of Cereal Science 81:108-117.

Secretaría de Economía (2002) NMX-FF-034/1-SCFI-2002. Productos Alimenticios no Industrializados para Consumo Humano-Cereales-Parte I: Maíz Blanco para Proceso Alcalino para Tortillas de Maíz y Productos de Maíz Nixtamalizado-Especificaciones y Métodos de prueba. Ciudad de México, México. 18 p.

SIAP, Servicio de Información Agroalimentaria y Pesquera (2018) Avance de siembras y cosechas. Resumen nacional por estado. http://infosiap.siap.gob.mx:8080/agricola_siap_gobmx/AvanceNacionalCultivo.do (Consultado Mayo, 2018).

Teklewold A., D. Wegary, A. Tadesse, B. Tadesse, K. Bantte, D. Friesen and M. Prasanna (2015) Quality Protein Maize. Guide to the Technology and its promotion in Ethiopia. CIMMYT. Ethiopia. $34 \mathrm{p}$.

Torres-Morales B., B. Coutiño-Estrada, A. Muñoz-Orozco, A. Santacruz-Varela, A. Mejía-Contreras, S. O. Serna-Saldivar, S. García-Lara y N. PalaciosRojas (2010) Selection for oil content in kernels of maize varieties of the Comiteco Race from Chiapas Mexico. Agrociencia 
44:679-689

Twumasi-Afriyie S., N. Palacios-Rojas, D. Friesen, A. Teklewold, D. Wegary, H. De Groote and B. M. Prasanna (2016) Guidelines for the Quality Control of Quality Protein Maize (QPM) Seed and Grain. CIMMYT: Technical Bulletin. Addis Ababa, Ethiopia. $45 \mathrm{p}$

Vázquez-Carrillo M. G., I. Rojas-Martinez, D. Santiago-Ramos, J. L. ArellanoVázquez, A. Espinosa-Calderón, M. García-Pérez and J. Crossa (2016) Stability analysis of yield and grain quality traits for the nixtamalization process of maize genotypes cultivated in the central high valleys of Mexico. Crop Science 56:3090-3099.

Vázquez-Carrillo M. G., D. Santiago-Ramos, M. Gaytán-Martínez, E. MoralesSánchez and M. J. Guerrero-Herrera (2015) High oil content maize: Physical, thermal and rheological properties of grain, masa, and tortillas. LWT-Food Science and Technology 60:156-161.

Vázquez-Carrillo M. G., D. Santiago-Ramos, Y. Salinas-Moreno, J. López-Cruz, M. C. Ybarra-Moncada y A. Ortega-Corona (2014) Genotipos de maíz (Zea mays L.) con diferente contenido de aceite y su relación con la calidad y textura de la tortilla. Agrociencia 48:159-172. 